\title{
Application of the predictive abdominal reoperation index for abdominal infection in patients with diagnosis of sepsis of the General Hospital of Mexico "Dr. Eduardo Liceaga"
}

\author{
Ansony R. Godinez-Vidal1*, Jose F. Chaga-Torres ${ }^{1}$, Christian I. Cruz-Romero ${ }^{1}$, Juan A. Villanueva-Herrero², \\ Billy Jimenez-Bobadilla ${ }^{2}$, Lisbeth Alarcón-Bernés ${ }^{1}$ and Noe I. Gracida-Mancilla ${ }^{1}$ \\ ${ }^{1}$ Departamento de Cirugía General, ${ }^{2}$ Departamento de Cirugía de Colon, Recto y Ano. Hospital General de México “Dr. Eduardo Liceaga”, Mexico City, \\ Mexico
}

\begin{abstract}
Background: Despite the improvement in care, recognition and management of multiple organ failure, availability of new antibiotics, advances in diagnostic techniques, and the decision to reintervention in a critically ill patient ultimately depend on a medical judgment. The use of the Abdominal Reoperation Predictive Index (ARPI) among patients with a possibility of reintervention allows the estimation of mortality being susceptible to be abated, reducing the time elapsed between the first operation and the relaparotomy, as well as a shorter stay in intensive care units. Objective: The objectives of this study were to determine the degree of certainty of the ARPI to diagnose the need for reoperation in patients with abdominal sepsis. Materials and Methods: An observational, descriptive, cross-sectional, retrospective study of patients from the General Hospital of Mexico "Eduardo Liceaga" belonging to the clinic of integral care of abdominal sepsis, from January to June 2016, with diagnosis of abdominal sepsis operated urgently by secondary peritonitis. Results: A total of 94 patients were included, $58.5 \%$ of male $(n=55)$ and $41.5 \%$ of female $(n=39)$, with an average age of 48.1 years (Standard mean [SD] \pm 17.4 ). The most frequently injured organ in the initial surgery was the cecal appendix in 39 cases (41.5\%) followed by the small intestine in 21 cases (22.3\%). Those cases with ARPI scores $>21$ were operated on 6 of 8 , corresponding to $75 \%$ with a score $>21$, while of the cases with ARPI score $<20$ points reoperated 23 of 86 , corresponding to $26.7 \%$ with a score $<20$ points. Fisher's exact test, $p=0.01$, statistically significant. Conclusions: The ARPI may be helpful in guiding the decision-making of laparotomy in patients diagnosed with secondary peritonitis.
\end{abstract}

Key words: Mortality. Laparotomy. Peritonitis.

\section{Introduction}

Secondary peritonitis is still an important cause of death in our time, so an early intervention could help reduce mortality. This problem cannot be modified without knowledge of the factors that affect the forecast.
After the initial laparotomy, a real challenge arises for the surgeon, in determining whether or not there is a secondary peritonitis in progress ${ }^{1,2}$ and whether a reoperation or not may be necessary for the survival of the patient ${ }^{3-5}$.

In the current practice, there is no consensus on the signs, symptoms, or laboratory values that are

\footnotetext{
${ }^{*}$ Correspondence:

Ansony R. Godinez-Vidal

E-mail: ansony.rgv@gmail.com

Available online: 21-03-2019 Rev Med Hosp Gen Mex. 2019;82(1):11-14 www.hospitalgeneral.mx 0185-1063/C 2018 Sociedad Médica del Hospital General de Mexico. Published by Permanyer México SA de CV. This is an open access article under the CC BY-NC-ND license (http://creativecommons.org/licenses/by-nc-nd/4.0/).
} 
indicative of a reoperation, so the decision to perform a relaparotomy should be based on consensus of medical opinions $\mathrm{s}^{6-8}$.

The use of the Abdominal Reoperation Predictive Index (ARPI) among patients with the possibility of reoperation allows us to estimate the mortality rate being susceptible to being reduced, decreasing the time elapsed between the first operation and relaparotomy, as well as a shorter stay in intensive care units 9 .

The precise and timely selection of patients for relaparotomy are a prerequisite for improving treatment in secondary peritonitis, so it is a priority to investigate more in this type of research leaving aside our surgical judgment based on the experiences combined even more with "hunches."

The aim of our study was to determine the degree of certainty of the ARPI to diagnose the need for reoperation in patients with abdominal sepsis.

\section{Materials and Methods}

An observational, descriptive, cross-sectional, retrospective study in patients from the General Hospital of Mexico "Dr. Eduardo Liceaga" belonging to the sepsis clinic and to the Comprehensive Care Clinic for abdominal sepsis, from January to June 2016, with diagnosis of abdominal sepsis emergency operated for secondary peritonitis. Characteristics of the initial surgery and parameters included in the ARPI were obtained, and the cases were followed up for 7 days postoperatively. The data were analyzed, considering as a variable the ARPI score and as a dependent variable the need for reoperation. The sample was divided into those cases that required reoperation and those that did not require; the mean ARPI score was obtained for each group and the differences between groups were validated using the Mann-Whitney U-test. Subsequently, taking the value 20 as the cutoff point, we proceeded to categorize the sample into two groups, one $>20$ and another below, and using a $2 \times 2$ table, the frequencies were observed, the exact test was applied. Fisher as a test statistic for statistical validation, and if statistical significance was obtained $(p<0.05)$, we proceeded to calculate the odds ratio, to determine the attributed risk.

\section{Results}

A total of 94 patients were included, $58.5 \%$ of male $(n=55)$ and $41.5 \%$ of female $(n=39)$, with an average age of 48.1 years (SD 4 17.4). The organs damaged in the initial surgery were the cecal appendix in 39 cases
Table 1. The distribution of the average ARPI score in reoperated and non-reoperated

\begin{tabular}{|l|c|c|}
\hline \multicolumn{3}{|c|}{ ARPI score for reoperated and non-reoperated } \\
\hline ARPI score & Reoperated & Non-reoperated \\
\hline Media & 12.03 & 7.54 \\
\hline Standard deviation & 9.22 & 6.25 \\
\hline Minimum & 3 & 3 \\
\hline Maximum & 30 & 23 \\
\hline Range & 27 & 20 \\
\hline
\end{tabular}

ARPI: Abdominal Reoperation Predictive Index.

(41.5\%), the small intestine in 21 cases $(22.3 \%)$, the colon in 15 cases (16\%), the bile duct in 8 cases $(8.5 \%)$, the uterus and its annexes in 5 cases (5.3\%), the stomach in 4 cases $(4.3 \%)$, and the pancreas in 2 cases $(2.1 \%)$.

Regarding the mechanism of damage, visceral perforation was the most common mechanism $(67 \%)$, followed by inflammation (19\%), obstruction (8\%), and ischemia (6\%). There was generalized peritonitis in $65 \%$ of the cases and located in $35 \%$. The fluid found in the first surgery was purulent in $59 \%$, intestinal fluid in $18 \%$, serous or citrine in $12 \%$, and blood in $3 \%$, and in $8 \%$, no fluid was found in the peritoneal cavity. Mortality for the sample was $15 \%$; $85 \%$ survived. Reoperation was required in $31 \%$ of the cases. When looking at the variables that make up the ARPI, and the frequency observed in the sample studied, there was persistent pain in 17 cases (18.1\%), ileus in 14 cases $(14.9 \%)$, discharge from the wound in 11 (11.7), alteration of wakefulness manifested by a Glasgow score of 12 or less in 6 cases $(6.4 \%)$, data of respiratory failure in 23 cases $(24.5 \%)$, and renal failure in 9 cases $(9.6 \%)$.

The distribution of the mean ARPI score in the reoperated subjects was 12.03 (SD \pm 9.22), and for the non-reoperated, there was a mean of $7.54(S D \pm 6.25)$ when we applied the Mann-Whitney statistic, $p=0.023$ statistically significant was obtained, table 1 .

When dividing the sample, we obtained that of those cases with ARPI score $>21,6$ of 8 cases were operated, which corresponds to $75 \%$ of the cases with a score $>21$; while in cases with an ARPI score of $<20$ points, 23 of 86 cases were reoperated and correspond to $26.7 \%$ of cases with $<20$ points. Fisher's exact test, $p=0.01$, statistically significant. When calculating the RM, a risk value of 8.21 was obtained with a $95 \%$ confidence interval for a range with a lower limit 
Table 2. Association between ARPI score (cutoff value: 21$)$ and need of reoperation

\begin{tabular}{|l|c|c|c|}
\multirow{2}{*}{ Variable } & \multicolumn{2}{|c|}{ Reoperation (\%) } & \multirow{2}{*}{ Total (\%) } \\
\cline { 2 - 3 } & Yes & No & \\
\hline ARPI score & \multicolumn{3}{|c|}{} \\
\hline$>21$ & $6(75.0)$ & $2(25.0)$ & $8(100.0)$ \\
\hline$<20$ & $23(26.7)$ & $63(73.3)$ & $86(100.0)$ \\
\hline Total & $29(30.9)$ & $65(69.1)$ & $94(100.0)$ \\
\hline
\end{tabular}

Cases with ARPI score $>21$ were operated in $75 \%$ of cases, while of the cases with ARPI score $<20$ points were reoperated in $26.7 \%$, ARPI: Abdominal Reoperation Predictive Index.

Table 3. Variables of the ARPI score with statistical significance in its association with the need for reoperation, persistent pain, ileus, and respiratory failure

\begin{tabular}{|l|l|c|}
\hline Variable & $\begin{array}{l}\text { Association with the } \\
\text { need of reoperation }\end{array}$ & $\boldsymbol{p}$-value \\
\hline Persistent pain & Yes & 0.006 \\
\hline Ileus & Yes & 0.021 \\
\hline Respiratory failure & Yes & 0.043 \\
\hline Secretion from the wound & No & 0.07 \\
\hline Disturbance of vigil & No & 0.6 \\
\hline Renal failure & No & 0.43 \\
\hline
\end{tabular}

ARPI: Abdominal Reoperation Predictive Index.

of 1.54 and an upper limit of 43.65 , which when not crossing the unit is considered valid, table 2 .

After analyzing each variable of the ARPI separately in its association with the need for reoperation, it was observed that persistent pain, ileus, and respiratory failure, if they showed a statistically significant difference $(p<0.05)$, while the secretion variables due to the wound, impaired wakefulness, and renal failure, they showed no association $(p>0.05)$, table 3 .

\section{Discussion}

At present, the decision to perform a relaparotomy in patients with secondary peritonitis continues to be based on "surgeon's judgment" despite the great inherent variability between them.

Surgical treatment of this condition focuses on the need to control the source of infection and abdominal lavage, for this, the preferred strategies to achieve these goals are relaparotomy on demand or planned, although no conclusive data have been determined in regarding morbidity and mortality in both strategies, the balance is tilted in favor of the demand strategy ${ }^{10}$.

However, we are aware that one of the main drawbacks of this strategy is the lack of criteria that help the surgeon to determine the opportune moment of a reoperation ${ }^{11-15}$.

Markers capable of indicating ongoing intra-abdominal infection have been investigated, it is known that multiorgan failure is strongly related to the result of peritonitis, it means, mortality ${ }^{16-18}$.

To date, the scoring systems available are not entirely applicable to patients with secondary peritonitis, so it is necessary to continue with the investigation of them. In one of his publications, $\mathrm{O}$ van Ruler mentions that 7 of 10 variables, which surgeons take into account to determine their therapy to follow, are unreliable as predictors of the need for relaparotomy ${ }^{19}$. In our study, analyzing each variable of the ARPI separately in its association with the need for reoperation, it was observed that three variables (persistent pain, ileus, and respiratory failure) showed a statistically significant difference $(p<0.05)$.

The ARPI was developed with the purpose of helping to decide if an abdominal reoperation should be performed in case of septic complications after surgery. This index was constructed as a proposal that integrated the clinical data most frequently presented in patients with post-operative peritonitis, subjected to a statistical validation by means of a multiple regression test. In its original presentation, it showed a diagnostic confidence $>88 \%$ when its value was $>15$ points, with which it was suggested that it is a tool that helps minimize subjectivity in the decision of the surgeon; however, its use was not widely disseminated or validated ${ }^{20}$.

In our hospital, post-operative peritonitis occurs in $38 \%$ of the cases of generalized peritonitis that is treated in the emergency department; for the present shows that $31 \%$ of the cases were reoperated.

When applying the ARPI to our sample, we found for the cases that required reoperation, an average score of 12 and for the non-reoperated a mean score of 7.5, with a statistically significant difference.

In the original proposal of Pusajó et $\mathrm{al}^{20}$., it was suggested that in cases with scores of $<20$, special studies were carried out to determine the need for reoperation, and in cases with scores $>20$, they were reoperated without further studies. We selected the score of 20 as 
a point of discrimination and obtained that of our cases with $>20$ points, $75 \%$ required reoperation, while those with scores $<20,26 \%$ required reoperation. Moreover, an 8 times higher risk of reoperation was obtained for patients with an ARPI score $>20$ points.

As mentioned by Pusajó et al., the systematic application of the index allows the quality of care to improve, the costs are lower, and the level of conflicts generated by the difficult decision to perform an operation is reduced ${ }^{20}$.

\section{Conclusions}

The ARPI can be of help in the orientation for the decision-making of relaparotomy in patients with diagnosis of secondary peritonitis. An ARPI score $>21$ raises the possibility of reintervention. Persistent pain, ileus, and respiratory failure are the three variables that we must consider as predictors of relaparotomy. The predictive index of abdominal reoperation is an excellent support tool for the surgeon to diagnose an infectious intra-abdominal complication, valid and easy to implement.

\section{Ethical disclosures}

Protection of human and animal subjects. The authors declare that no experiments were performed on humans or animals for this study.

Confidentiality of data. The authors declare that they have followed the protocols of their work center on the publication of patient data.
Right to privacy and informed consent. The authors declare that no patient data appear in this article.

\section{References}

1. Demmel N, Maag K, Osterholzer G. The value of clinical parameters for determining the prognosis of peritonitis-validation of the Mannheim peritonitis index. Langenbecks Arch Chir. 1994;379:152-8.

2. Bracho-Riquelme R, Melero-Vela A, Ramirez AT. Mannheim peritonitis index validation study at the hospital general de durango (Mexico). Cir Cir. 2002;70:217-25.

3. Kiewiet JJ, van Ruler O, Boermeester MA, Reitsma JB. A decision rule to aid selection of patients with abdominal sepsis requiring a relaparotomy. BMC Surg. 2013;13:28.

4. Berger D, Buttenschoen K. Management of abdominal sepsis. Langenbecks Arch Surg. 1998;383:35-43.

5. Bosscha K, van Vroonhoven TJ, van der Werken C. Surgical management of severe secondary peritonitis. Br J Surg. 1999;86:1371-7.

6. Cheadle WG, Spain DA. The continuing challenge of intra-abdominal infection. Am J Surg. 2003;186:15S-22.

7. van Ruler O, Mahler CW, Boer KR, et al. Comparison of on-demand vs planned relaparotomy strategy in patients with severe peritonitis: a randomized trial. JAMA. 2007;298:865-72.

8. Schein M. Surgical management of intra-abdominal infection: is there any evidence? Langenbecks Arch Surg. 2002;387:1-7.

9. Van Goor H. Interventional management of abdominal sepsis: when and how. Langenbecks Arch Surg. 2002;387:191-200.

10. Novotny AR, Emmanuel K, Hueser N, et al. Procalcitonin ratio indicates successful surgical treatment of abdominal sepsis. Surgery. 2009;145:20-6.

11. Hill BA, Yamagichi K, Flynn JJ, Miller DR. Diagnostic sonography in general surgery. Arch Surg. 1975;110:1089-94.

12. Posteraro $\mathrm{RH}$, Ravin C. Magnetic resonance imaging in critically ill patients. Crit Care Med. 1987:9:273-84.

13. Civetta JM. The inverse relationship between cost and survival. J Surg Res. 1987; 14:265-70.

14. Cerda C. Manejo quirúrgico de la sepsis abdominal. Cir Gen. 2011;33 Supl 1:S25-7.

15. Pérez JC. Sepsis abdominal. Rev Assoc Mex Med Crít Ter Intensiva. 2002;16:124-35.

16. Lamme B, Boermeester MA, Reitsma JB, et al. Meta-analysis of relaparotomy for secondary peritonitis. Br J Surg. 2002;89:1516-24.

17. Hinsdale JG, Jaffe BM. Re-operation for intra-abdominal sepsis. Indications and results in modern critical care setting. Ann Surg. 1984;199:31-6.

18. Machiedo GW, Tikellis J, Suval W, et al. Reoperation for sepsis. Am Surg. 1985;51:149-54

19. van Ruler O, Lamme B, de Vos R, et al. Decision making for relaparotomy in secondary peritonitis. Dig Surg. 2008;25:339-46.

20. Pusajó JF, Bumaschny E, Doglio GR, et al. Postoperative intra-abdominal sepsis requiring reoperation. Value of a predictive index. Arch Surg. 1993;128:218-22. 